\title{
Noncollinear antiferromagnetic states in Ru-based Heusler compounds induced by biquadratic coupling
}

\author{
Eszter Simon $\odot,{ }^{1, *}$ Andreas Donges, ${ }^{2}$ László Szunyogh,,${ }^{1,3}$ and Ulrich Nowak $\oplus^{2}$ \\ ${ }^{1}$ Department of Theoretical Physics, Budapest University of Technology and Economic, Budafoki út 8, H-1111 Budapest, Hungary \\ ${ }^{2}$ Department of Physics, University of Konstanz, D-78464 Konstanz, Germany \\ ${ }^{3}$ MTA-BME Condensed Matter Research Group, Budapest University of Technology and Economics, \\ Budafoki út 8, H-1111 Budapest, Hungary
}

(Received 22 December 2019; revised 5 May 2020; accepted 29 July 2020; published 17 August 2020)

\begin{abstract}
We investigate the magnetic properties of $\mathrm{Ru}_{2} \mathrm{MnZ}(Z=\mathrm{Sn}, \mathrm{Sb}, \mathrm{Ge}, \mathrm{Si})$ chemically ordered, full Heusler compounds for zero as well as finite temperatures. Based on first-principles calculations we derive the interatomic isotropic bilinear and biquadratic couplings between $\mathrm{Mn}$ atoms from the paramagnetic state. We find frustrated isotropic couplings for all compounds and, in the case of $Z=\mathrm{Si}$ and $\mathrm{Sb}$, a nearest-neighbor biquadratic coupling that favors perpendicular alignment between the Mn spins. By using an extended classical Heisenberg model in combination with spin dynamics simulations we obtain the magnetic equilibrium states. From these simulations we conclude that the biquadratic coupling, in combination with the frustrated isotropic interactions, leads to noncollinear magnetic ground states in the $\mathrm{Ru}_{2} \mathrm{MnSi}$ and $\mathrm{Ru}_{2} \mathrm{MnSb}$ compounds. In particular, for these alloys we find two distinct, noncollinear ground states which are energetically equivalent and can be identified as $3 Q$ and $4 Q$ states on a frustrated fcc lattice. Investigating the thermal stability of the noncollinear phase we find that, in the case of $\mathrm{Ru}_{2} \mathrm{MnSi}$, the multiple- $Q$ phase undergoes a transition to the single $Q$ phase, while in case of $\mathrm{Ru}_{2} \mathrm{MnSb}$ the corresponding transition is not obtained due to the larger magnitude of the nearest-neighbor biquadratic coupling.
\end{abstract}

DOI: 10.1103/PhysRevMaterials.4.084408

\section{INTRODUCTION}

Technological and fundamental interest in the antiferromagnetic (AFM) materials is increasingly growing since materials with novel-type antiferromagnetic structures are possible candidates for a new generation of spintronic devices [1-3]. Antiferromagnetic materials can complement or even replace ferromagnetic (FM) components in spintronic devices with improved properties due to their enhanced stability against the perturbation via external magnetic fields. Many technologically important effects have already been implemented using an AFM material as the main element of the system, such as the ultrafast spin dynamics, magnetotransport, or exchange bias effects [4-8].

AFM Heusler alloys can be a possible extension of the class of known antiferromagnetic materials, but relatively few AFM Heusler alloys are known with sufficiently high Néel temperatures [9]. Full Heusler compounds, with the chemical formula $X_{2} Y Z$, where $X$ and $Y$ are transition metals and $Z$ is a $p$ group element, are mostly ferromagnetic, but it can be transformed from the FM to the AFM state by changing the atomic composition. In the case of the $\mathrm{Ni}_{2} \mathrm{MnAl}$ alloy, for instance, if $\mathrm{Mn}$ atoms are also placed on the $\mathrm{Al}$ sites, these $\mathrm{Mn}$ atoms interact antiferromagnetically with the nearest-neighbor (NN) $\mathrm{Mn}$ atoms on the original sites and this material becomes a compensated antiferromagnet in the fully disordered (B2)

\footnotetext{
*esimon@phy.bme.hu
}

state [10,11], a phase which is called structurally induced antiferromagnetism [12]. In $\mathrm{Ru}_{2} \mathrm{MnZ}(Z=\mathrm{Sn}, \mathrm{Sb}, \mathrm{Ge}, \mathrm{Si}$ ) alloys the formation of antiferromagnetic order is not structurally induced. From earlier theoretical and experimental work it is known that the magnetic ground state of the $\mathrm{Ru}_{2} \mathrm{MnZ}$ series of Heusler alloys corresponds to the second kind of AFM order or simply AF2 $[13,14]$. This magnetic state was also confirmed by a recent first-principles calculation, in which it was shown that the chemical disorder significantly reduced the transition temperature of the $\mathrm{Ru}_{2} \mathrm{MnSi}$ system [15]. The crystal structure of the ordered $\mathrm{Ru}_{2} \mathrm{MnZ}$ is $L 2_{1}$ type, where the Mn atoms fully occupy one of four interpenetrating fcc subblatices of the $L 2_{1}$ structure. In Ref. [15] it was reported that the competition between the strong antiferromagnetic nextnearest-neighbor (NNN) exchange coupling and the weakly ferromagnetic $\mathrm{NN}$ exchange coupling, results in the second type AFM ordering. In this AFM state, illustrated in Fig. 1, the magnetic atoms on neighboring (111) planes are coupled antiparallel, while atoms within such a (111) plane couple ferromagnetically, leading to a frustration of the ferromagnetic $\mathrm{NN}$ exchange.

Frustration in the magnetic structure can originate from geometric frustration of a lattice or from the competition between NN and NNN or even farther exchange interactions. Geometrical frustration of a spin configuration is known for triangular antiferromagnets [16] or in highly correlated metals [17]. Frustration with higher-order exchange terms can lead to various exotic magnetic states such as the multiple $-Q$ spin order, where the spin texture is characterized by a mul- 


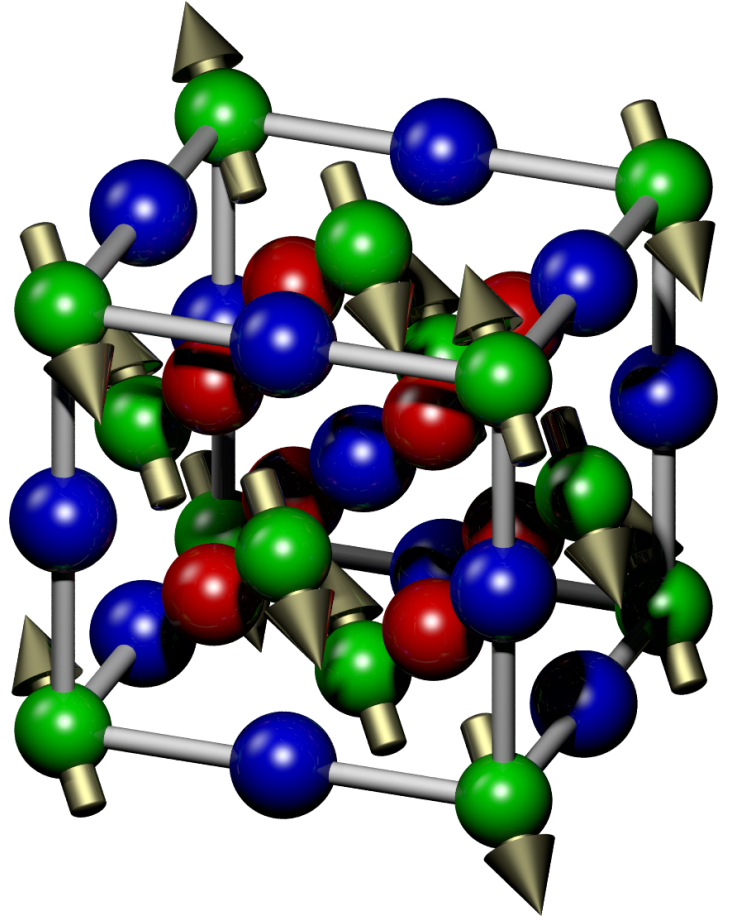

FIG. 1. Unit cell of the $\mathrm{Ru}_{2} \mathrm{MnZ}$ full Heusler compound, with $\mathrm{Ru}$ atoms in red, $\mathrm{Mn}$ atoms in green, and $Z$ atoms in blue. Golden arrows show the second kind of antiferromagnetic order (AF2) on the fcc sublattice of $\mathrm{Mn}$ atoms.

tiple number of coexisting magnetic modulation $Q$ vectors. A $3 Q$ state stabilized by four-spin interaction was obtained from model simulations on a triangular lattice [18-21] as well as using $a b$ initio calculations in magnetic thin film systems [22] and in itinerant hexagonal magnets [23,24]. In the case of the highly correlated Kondo lattice compound $\mathrm{UCu}_{5}$ it was shown that a stable $4 Q$ state is formed at zero temperature due to the biquadratic coupling [25]. Note that the AF2 state visualized in Fig. 1 corresponds to the $1 Q$ state with $Q=(1,1,1)$.

In Ref. [15] it was mentioned that the biquadratic coupling lifts the degeneracy of the highly frustrated AF2 states and positive/negative $\mathrm{NN}$ biquadratic couplings can lead to collinear/noncollinear magnetic ground states in the case of $\mathrm{RuMn}_{2}(\mathrm{Sn}, \mathrm{Ge})$ and $\mathrm{RuMn}_{2}(\mathrm{Sb}, \mathrm{Si})$, respectively. However, the main focus of Ref. [15] was on the Néel temperature of these Heusler alloys which was hardly affected by the weak biquadratic couplings, while the effect of chemical disorder on the spin structure and the Néel temperature was investigated in detail using Monte Carlo simulations.

In this paper we examine the effect of the biquadratic coupling on the magnetic state of the chemically ordered $\mathrm{Ru}_{2} \mathrm{MnZ}$ Heusler compounds using atomistic spin model simulations. The isotropic bilinear and biquadratic couplings are determined from the paramagnetic state using first-principles calculations. Based on these spin model parameters we employ an extended, classical Heisenberg model and explicitly demonstrate that biquadratic couplings result in a noncollinear magnetic ground state in the case of $\mathrm{Ru}_{2} \mathrm{MnSi}$ and $\mathrm{Ru}_{2} \mathrm{MnSb}$ compounds, where the $\mathrm{NN}$ biquadratic coupling favors perpendicular alignment between the Mn moments. In these cases we carefully analyze the spin configurations obtained at zero temperature and conclude that $3 Q$ and $4 Q$ states occur with equal probability as the energy of these states remain degenerate within the applied model. We also show that, depending on the magnitude of the biquadratic coupling, at finite temperature the noncollinear magnetic phase can transform to the $1 Q$ phase.

\section{CALCULATION DETAILS}

We performed self-consistent calculations for the $\mathrm{Ru}_{2} \mathrm{MnZ}$ compounds within the local spin-density approximation (LSDA) [26] by using the screened Korringa-Kohn-Rostoker (KKR) method $[27,28]$ in the atomic sphere approximation (ASA) by expanding the partial waves up to $l_{\max }=3(s p d f$ basis) inside the atomic spheres. The electronic structure was determined in the paramagnetic state in terms of the scalar relativistic disordered local moment (DLM) scheme [29]. For all investigated alloys we used the experimental lattice constants $(a)$ of the $L 2_{1}$ lattice structure [15].

First-principles descriptions of itinerant magnetism mostly rely on the adiabatic decoupling of the fast electron hopping and the slow transversal motion of spins. As a further simplification of the theory longitudinal spin-fluctuations are often neglected and the so-called rigid spin approximation is used. The spin-cluster expansion (SCE) technique developed by Drautz and Fähnle provides a systematic parametrization of the adiabatic magnetic energy [30,31]. The crucial step of the SCE is the evaluation of orientational averages restricted to fixed spin-configurations of selected clusters. These restricted averages can be efficiently performed using the coherentpotential (mean-field) approximation implemented within the DLM scheme. Therefore, to derive spin model parameters for the $\mathrm{Ru}_{2} \mathrm{MnZ}$ compounds we employed the SCE technique as combined with the DLM method as described in Ref. [32].

Our first-principles calculations result in a classical spin Hamiltonian of the form

$$
H=-\frac{1}{2} \sum_{i, j} J_{i j} \vec{s}_{i} \cdot \vec{s}_{j}-\frac{1}{2} \sum_{i, j} B_{i j}\left(\vec{s}_{i} \cdot \vec{s}_{j}\right)^{2},
$$

where $\vec{s}_{i}$ is the unit vector along the direction of the spin moment of atom $i$. In Eq. (1) the first term corresponds to a Heisenberg model where $J_{i j}$ is the isotropic exchange interaction. The second term describes the isotropic biquadratic interaction between spins $i, j$ with the coupling constants $B_{i j}$. In the sign convention of Eq. (1), $J_{i j}>0$ describes the ferromagnetic coupling between the magnetic moments, while $J_{i j}<0$ corresponds to the antiferromagnetic interaction. Likewise, $B_{i j}>0$ favors collinear and $B_{i j}<0$ perpendicular alignment of neighboring Mn spins. These spin model parameters were calculated for 369 neighbors within a radius of $3 a$, where $a$ is the lattice constant of the corresponding system.

To study the magnetic ground state of the system and the equilibrium state at finite temperatures, we solved the stochastic Landau-Lifshitz-Gilbert (SLLG) equation on a discrete lattice

$$
\frac{\partial \vec{s}_{i}}{\partial t}=-\frac{\gamma}{\left(1+\alpha^{2}\right) \mu_{\mathrm{s}}} \vec{s}_{i} \times\left(\vec{H}_{i}+\alpha \vec{s}_{i} \times \vec{H}_{i}\right),
$$


by means of Langevin dynamics, using a Heun algorithm [33,34]. The SLLG equation includes the gyromagnetic ratio $\gamma$, a phenomenological damping parameter $\alpha$, and the effective field

$$
\vec{H}_{i}=\vec{\zeta}_{i}(t)-\frac{\partial H}{\partial \vec{s}_{i}}=\vec{\zeta}_{i}(t)+\sum_{j(\neq i)} J_{i j} \vec{s}_{j}+2 \sum_{j(\neq i)} B_{i j}\left(\vec{s}_{i} \cdot \vec{s}_{j}\right) \vec{s}_{j},
$$

which considers the influence of a temperature $T$ by adding a stochastic noise term $\vec{\zeta}_{i}(t)$, obeying the properties of white noise [35]

$$
\begin{gathered}
\left\langle\vec{\zeta}_{i}(t)\right\rangle=0, \\
\left\langle\zeta_{i}^{\eta}(t) \zeta_{j}^{\theta}\left(t^{\prime}\right)\right\rangle=\frac{2 k_{B} T \alpha \mu_{s}}{\gamma} \delta_{i j} \delta_{\eta \theta} \delta\left(t-t^{\prime}\right) .
\end{gathered}
$$

Here $i, j$ denote lattice sites and $\eta$ and $\theta$ Cartesian components of the stochastic noise.

We used a 884736 atoms in the unit cell of the Mn sublattice with periodic boundary conditions and the couplings included up to the sixth NN shell. Two kinds of simulations were performed: To check the magnetic ground state we cooled the system down slowly, starting from the paramagnetic state using $\alpha=0.0001$. For calculating the specific heat, this parameter was set to 0.5 , and the system was heated up incrementally, starting from the previously determined ground-state spin configuration.

\section{RESULTS}

\section{A. Spin-model parameters}

First we performed first-principles electronic structure calculations for the $\mathrm{Ru}_{2} \mathrm{MnZ}$ series of the full Heusler compounds in the $L 2_{1}$ geometry, where the electronic structure was determined in the paramagnetic state. Similarly as in Ref. [15], we also found that the smallest local magnetic moment of $\mathrm{Mn}$ has the $\mathrm{Ru}_{2} \mathrm{MnSi}$ system with value of $2.90 \mu_{B}$, while for $\mathrm{Ru}_{2} \mathrm{MnSb}$ the largest $\mathrm{Mn}$ magnetic moment were obtained with value of $3.55 \mu_{B}$. From the selfconsistent potentials we determined the isotropic bilinear and biquadratic couplings between the $\mathrm{Mn}$ atoms for the $\mathrm{Ru}_{2} \mathrm{MnZ}$ compounds employing the spin-cluster expansion technique. Note that, according to the definition of the spin Hamiltonian in Eq. (1), our spin-model parameters are twice as large than those in Ref. [15]. The NN isotropic exchange coupling is ferromagnetic for all cases and smaller in magnitude than the antiferromagnetic second $\mathrm{NN}$ interaction as can be inferred from Fig. 2(a). The third NN coupling is almost vanishing, while the fourth $\mathrm{NN}$ interaction is ferromagnetic again and the magnitude of the couplings become negligible beyond the fourth $\mathrm{NN}$ shell.

In Fig. 2(b) the biquadratic couplings are also presented as a function of the distance between the Mn atoms. The NN biquadratic coupling is positive for $\mathrm{Ru}_{2} \mathrm{MnGe}$ and $\mathrm{Ru}_{2} \mathrm{MnSn}$, while negative for $\mathrm{Ru}_{2} \mathrm{MnSi}$ and $\mathrm{Ru}_{2} \mathrm{MnSb}$; and beyond the second NN biquadratic coupling, the $B_{i j}$ decays rapidly. According to the sign convention in Eq. (1), negative $B_{i j}$ means that the favored configuration between the Mn moments is perpendicular.

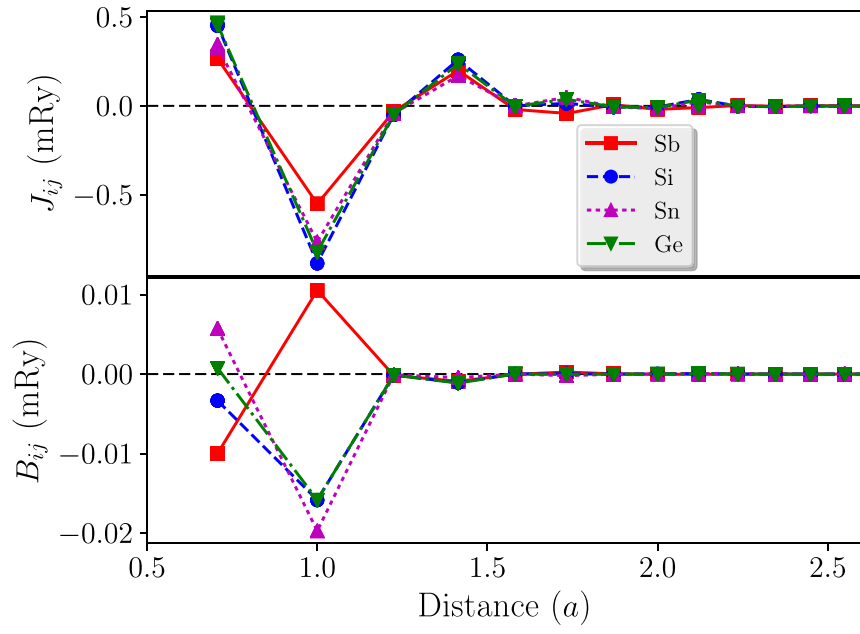

FIG. 2. Calculated Mn-Mn exchange interactions, $J_{i j}$ and biquadratic couplings, $B_{i j}$ for $\mathrm{Ru}_{2} \mathrm{MnZ}(Z=\mathrm{Sn}, \mathrm{Si}, \mathrm{Sb}, \mathrm{Ge})$ alloys as a function of distance between $\mathrm{Mn}$ atoms in unit of the lattice constant $a$.

\section{B. Magnetic ground state}

In agreement with previous theoretical results $[14,15]$, the ferromagnetic $\mathrm{NN}$ and antiferromagnetic $\mathrm{NNN}$ exchange interactions presented in Fig. 2 strongly indicate an AF2 magnetic ground state [36] for all investigated alloys. As discussed in Ref. [37] there are several equivalent AF2 structures that are linear combinations of the collinear AF2 states depicted in Fig. 1 related to the four possible wave vectors: $\vec{Q}_{1}=$ $\frac{\pi}{a}(1,1,1), \vec{Q}_{2}=\frac{\pi}{a}(-1,1,1), \vec{Q}_{3}=\frac{\pi}{a}(1,-1,1)$, and $\vec{Q}_{4}=$ $\frac{a}{a}(1,1,-1)$, with $a$ being the lattice constant of the fcc lattice. Such spin configurations can be described as multiple $-Q$ states,

$$
\vec{s}_{i}=\frac{1}{\sqrt{M}} \sum_{n=1}^{M} \vec{s}_{n}^{M} \exp \left(i \vec{Q}_{n}^{M} \vec{R}_{i}\right),
$$

where $M \in\{1,2,3,4\}, \vec{Q}_{n}^{M} \in\left\{\vec{Q}_{1}, \vec{Q}_{2}, \vec{Q}_{3}, \vec{Q}_{4}\right\}$, and $\vec{R}_{i}$ stands for the lattice vector of site $i$. The generating spin vectors $\vec{s}_{n}^{M}$ of unit length should be determined such that $\vec{s}_{i}$ will also be unit vectors. From this condition it can easily be deduced that a $2 Q$ structure $(M=2)$ is described by four magnetic sublattices associated with four neighboring sites of the fcc lattice forming a regular tetrahedron, with spins being aligned either antiparallel or normal to each other. Both the $3 Q(M=$ $3)$ and the $4 Q(M=4)$ textures can be described by doubling this magnetic unit cell, thus by eight magnetic sublattices. In one tetrahedron the spin vectors make an angle of either $109.5^{\circ}$ (tetrahedral angle) or $70.5^{\circ}\left(=180^{\circ}-109.5^{\circ}\right)$, i.e., $\vec{s}_{i}$. $\vec{s}_{j}= \pm 1 / 3(i \neq j)$, while the spins in the other tetrahedron are reversed as compared to the first one. One important difference between these two states is that for a $3 Q$ state one can always find a (111) type of plane which is magnetically compensated, while in a $4 Q$ state the spins in each tetrahedra compensate each other, thus, up to a global rotation of the spin vectors, the $4 Q$ state restores the cubic symmetry [37].

An effective isotropic spin model for the eight magnetic sublattices can easily be set up by making use of the cubic symmetry of the underlying fcc lattice. Let us denote the 
sublattices corresponding to the two tetrahedral unit cells by $a$, $b, c, d$ and $A, B, C, D$, where the sites in the sublattices labeled by the same small and capital letter are shifted by $a(n, m, k)$ with $n, m, k$ being integers. The corresponding bilinear spin model

$$
H_{\mathrm{bl}}=-\frac{1}{2} \sum_{\alpha, \beta} J_{\alpha \beta} \vec{s}_{\alpha} \cdot \vec{s}_{\beta},
$$

with $\alpha$ and $\beta$ labeling sublattices, contains only three independent parameters: $J_{\mathrm{aa}}, J_{\mathrm{aA}}$, and $J_{\mathrm{ab}}$ that refer to the effective intrasublattice interaction, to the interaction between the same kind of sublattices in the two tetrahedra and to the interaction between different kinds of sublattices, respectively. In the fourth $\mathrm{NN}$ approach, these interactions can be expressed as

$$
J_{\text {aa }}=6 J_{4}, \quad J_{\mathrm{aA}}=6 J_{2}, \quad J_{\mathrm{ab}}=2 J_{1}+4 J_{3},
$$

where $J_{n}$ denotes the atomic exchange parameters between the $n$th neighbors. It is then simple to show that in the billinear model all the multiple $-Q$ states discussed above have the same energy, $E_{\mathrm{AF} 2}=\frac{1}{2}\left(J_{\mathrm{aA}}-J_{\mathrm{aa}}\right)$ as normalized to one magnetic atom.

By including biquadratic couplings into the sublattice spin model

$$
H_{\mathrm{bq}}=-\frac{1}{2} \sum_{\alpha, \beta} B_{\alpha \beta}\left(\vec{s}_{\alpha} \cdot \vec{s}_{\beta}\right)^{2},
$$

the coupling coefficients $B_{\alpha \beta}$ have the same structure as mentioned in the context of Eq. (8). The biquadratic interactions change the energy of the AF2 states by

$$
\begin{aligned}
& E_{\mathrm{bq}}^{1 Q}=-\frac{1}{2}\left(B_{\mathrm{aa}}+B_{\mathrm{aA}}+6 B_{\mathrm{ab}}\right), \\
& E_{\mathrm{bq}}^{2 Q}=-\frac{1}{2}\left(B_{\mathrm{aa}}+B_{\mathrm{aA}}+2 B_{\mathrm{ab}}\right),
\end{aligned}
$$

and

$$
E_{\mathrm{bq}}^{3 Q}=E_{\mathrm{bq}}^{4 Q}=-\frac{1}{2}\left(B_{\mathrm{aa}}+B_{\mathrm{aA}}+\frac{2}{3} B_{\mathrm{ab}}\right) .
$$

This means that the biquadratic coupling between different kinds of sublattices $B_{\mathrm{ab}}$ lifts the degeneracy of the AF2 states: if $B_{\mathrm{ab}}>0$ then the collinear $1 Q$ states will be the ground state and for $B_{\mathrm{ab}}<0$ the noncollinear $3 Q$ and $4 Q$ states have the lowest energy. The $2 Q$ state will always be of higher energy than either the $3 Q, 4 Q$, or the $1 Q$ state, and is therefore not expected to be observed. It should be noted that it is primarily the NN biquadratic coupling $B_{1}$, which makes a difference in the energy of the AF2 states since $B_{\mathrm{ab}} \sim 2 B_{1}$, while the NNN biquadratic coupling $B_{2}$ is irrelevant in lifting the degeneracy.

From the calculated spin-model parameters we determined the magnetic ground state of the chemically ordered $\mathrm{Ru}_{2} \mathrm{MnZ}$ Heusler compound via simulated annealing. In all cases, the obtained configurations are characterized by alternating (111) planes with reversed spin configurations, stemming from the strong antiferromagnetic NNN exchange interaction. According to the considerations above, we found that depending on the sign of $B_{1}$ different antiferromagnetic magnetic ground states were formed. As shown in Fig. 2 in case of the $\mathrm{Ru}_{2} \mathrm{MnGe}$ and $\mathrm{Ru}_{2} \mathrm{MnSn}$ compounds, the $\mathrm{NN}$ biquadratic coupling, $B_{1}$ is positive, while for the $\mathrm{Ru}_{2} \mathrm{MnSi}$ and $\mathrm{Ru}_{2} \mathrm{MnSb}$ alloys it is negative. For $B_{1}>0$, i.e., for $Z=\mathrm{Sn}$ and $\mathrm{Ge}$,

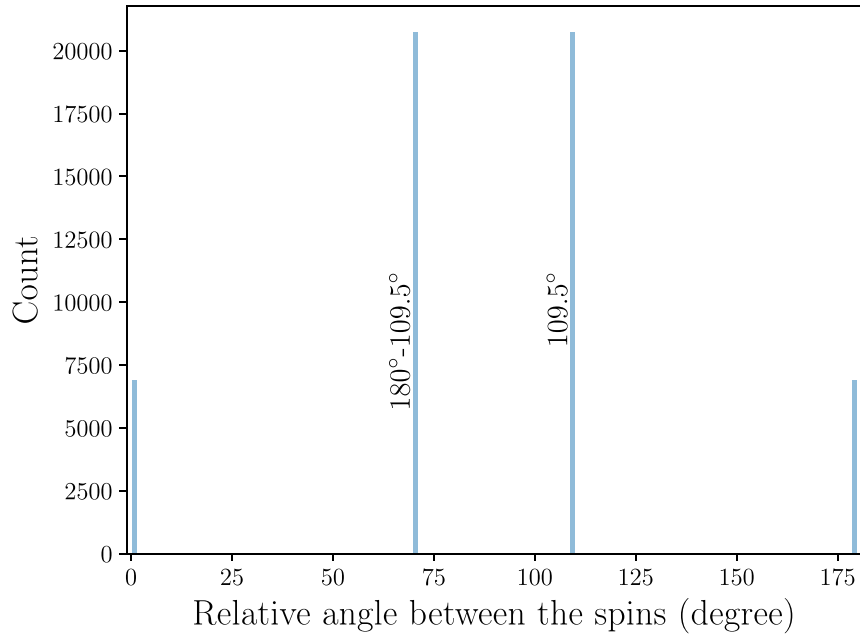

FIG. 3. Distribution of the angles between an arbitrarily chosen spin $\vec{s}_{i}$ and the remaining lattice spins $\vec{s}_{j \neq i}$, in the case of negative nearest-neighbor biquadratic coupling. Beyond the usual $0^{\circ}$ and $180^{\circ}$ angles, other tetrahedral, $109.5^{\circ}$ and $180^{\circ}-109.5^{\circ}$ angles appear as well due to the biquadratic coupling.

all spins are coupled ferromagnetically on each (111) plane, leading to an energetically favored collinear $1 Q$ state, as it is also observed in other fcc-AFMs such as the metal oxides $M \mathrm{O}(M=\mathrm{Mn}, \mathrm{Fe}, \mathrm{Co}, \mathrm{Ni})$ for instance [38]. In the case of $B_{1}<0$, i.e., for $Z=\mathrm{Si}$ and $\mathrm{Sb}$, the magnetic order in the (111) plane is more complicated, as neighboring spins want to align perpendicular to each other.

We calculated the distribution of the relative angles between the spin moments in the simulated cell of spin configuration and found that, beyond $0^{\circ}$ and $180^{\circ}$, relative angles of $109.5^{\circ}$ and $70.5^{\circ}$ occur in the system as demonstrated in Fig. 3. This distribution obviously corresponds to the $3 Q$ and $4 Q$ states displayed in the upper and lower panels of Fig. 4,
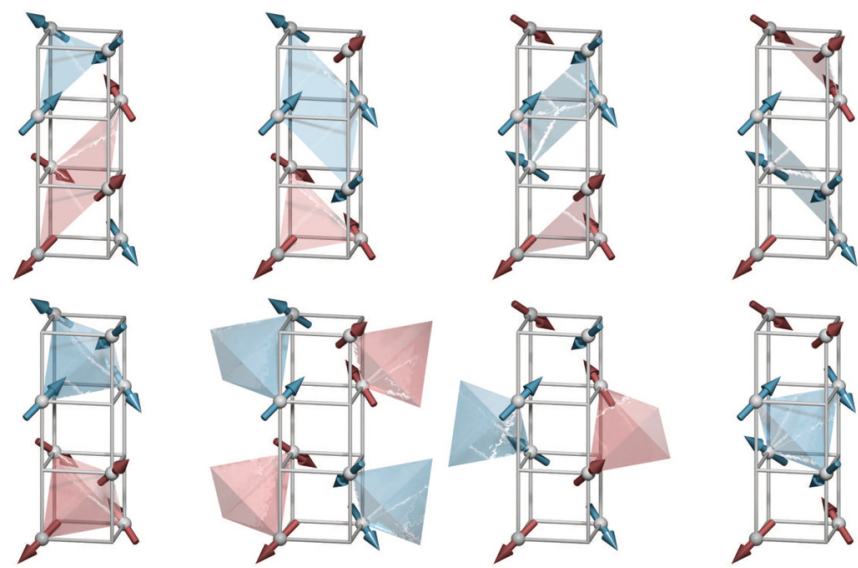

FIG. 4. Magnetic ground-state configurations simulated for $\mathrm{Ru}_{2} \mathrm{MnZ}(Z=\mathrm{Si}, \mathrm{Sb})$ Heusler compounds with negative $\mathrm{NN}$ biquadratic coupling $B_{1}$. The upper panel shows different variations of $3 Q$ states, where the fully compensated (111) planes are indicated with red and blue colors. In the lower panel, different variations of $4 Q$ states can be seen, with fully compensated tetrahedra indicated by red and blue colors. 


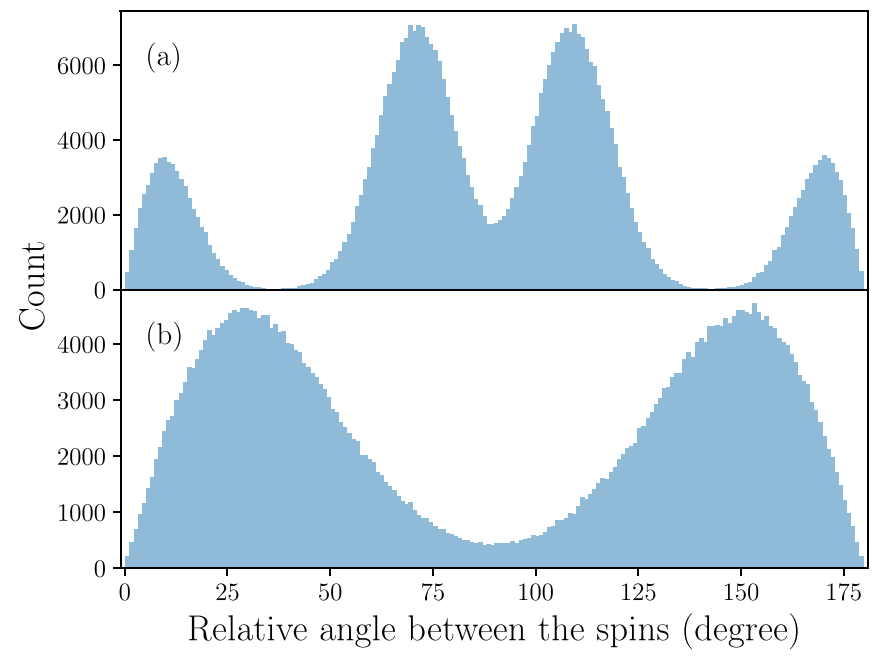

FIG. 5. Distribution of the angle between an arbitrarily chosen spin $\vec{s}_{i}$ and the remaining lattice spins $\vec{s}_{j \neq i}$ for the $\mathrm{Ru}_{2} \mathrm{MnSi}$ system at two different temperatures. At (a) $T=20 \mathrm{~K}$ the $4 Q$ state is still stable, while at (b) $T=214 \mathrm{~K}$ antiferromagnetic $1 Q$ state is formed.

respectively. From these figures it can be inferred that by fixing an arbitrary spin in a magnetic unit cell, there is one spin aligned antiparallel to this spin, while three spins make $109.5^{\circ}$ and three spins make $70.5^{\circ}$ with the chosen spin. This explains the ratios of 1:3:3:1 for the occurrence of the four relative angles in Fig. 3.

For the case of $B_{1}<0$ we determined the probability of the $3 Q$ and $4 Q$ states in the ground state by relaxing a total of 512 random configurations of $48^{3}$ spins. We detected $3 Q$ states in 253 cases $(49.4 \%)$, while $4 Q$ states occured in 259 cases $(50.6 \%)$. These simulational results indicate that the magnetic ground state of the system corresponds to an ensemble of $3 Q$ and $4 Q$ states of equal probability.

We also calculated the difference between the energy of the ground-state configurations and that of the collinear $1 Q$ state and obtained $\Delta E_{\mathrm{Si}}=-0.0096 \mathrm{mRy} / \mathrm{spin}$ and $\Delta E_{\mathrm{Sb}}=$ $-0.0275 \mathrm{mRy} / \mathrm{spin}$, respectively. These values are in good agreement with the analytical expressions in Eqs. (10) and (12), implying $E_{\mathrm{bl}}^{3 Q}-E_{\mathrm{bl}}^{1 Q}=\frac{8}{3} B_{a b} \sim \frac{16}{3} B_{1}$, which gives $-0.0089 \mathrm{mRy} / \mathrm{spin}$ for $\mathrm{Si}$ and $-0.0266 \mathrm{mRy} / \mathrm{spin}$ for $\mathrm{Sb}$.

To estimate the importance of relativistic effects not present in the model (1), we performed calculations of the cubic anisotropy constant $K$ for $\mathrm{Ru}_{2} \mathrm{MnSi}$ in the $1 Q$ state and obtained a value of $K=(5 \pm 1) \times 10^{-8}$ Ryd. Since this value is by about two orders smaller in magnitude than the biqudratic couplings, the effect of the cubic anisotropy can indeed be neglected in our present calculations.

\section{Finite temperature simulations}

Next, we investigate the equilibrium phases of the $\mathrm{Ru}_{2} \mathrm{MnSi}$ and $\mathrm{Ru}_{2} \mathrm{MnSb}$ compounds at finite temperatures. As it was demonstrated in Fig. 3 in terms of relative angles, the magnetic ground state of these systems is noncollinear due to the negative NN biquadratic coupling. For the case of $\mathrm{Ru}_{2} \mathrm{MnSi}$, the distribution of the relative angles between the spins at two temperatures is shown in Fig. 5. From Fig. 5(a)

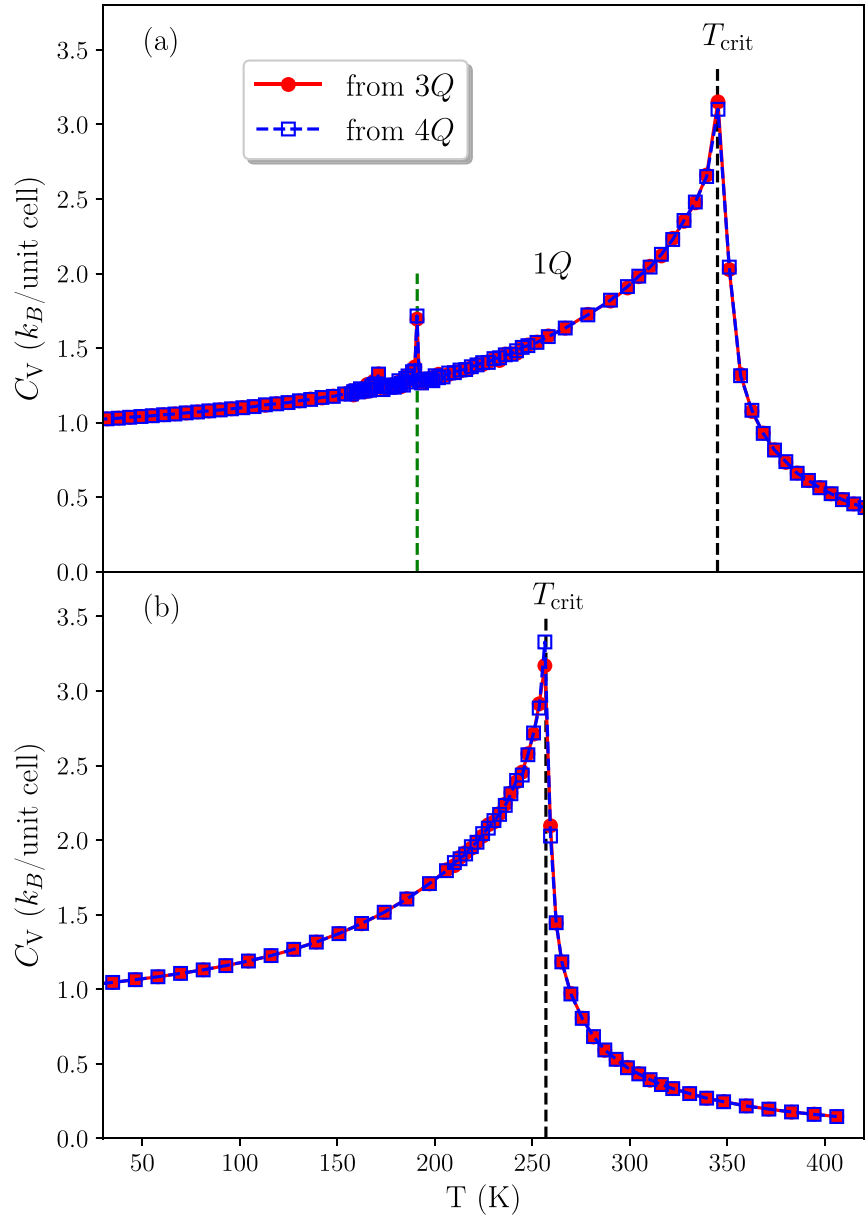

FIG. 6. Calculated heat capacity as a function of temperature for the chemically ordered full Heusler compounds (a) $\mathrm{Ru}_{2} \mathrm{MnSi}$ and (b) $\mathrm{Ru}_{2} \mathrm{MnSb}$. In both cases, the simulations were started at $0 \mathrm{~K}$ from either the $3 Q$ or the $4 Q$ configurations and no difference in the thermal behavior of the systems was detected. The dashed lines denote the phase transitions. In the case of $\mathrm{Ru}_{2} \mathrm{MnSi}$ the multiple $-Q$ state transforms into the antiferromagnetic $1 Q$ state, then to the paramagnetic state. In the case of $\mathrm{Ru}_{2} \mathrm{MnSb}$ the first phase transition is missing and the multiple- $Q$ states transforms directly to the paramagnetic state.

it is obvious that at $T=20 \mathrm{~K}$ the noncollinear state remains stable as the distribution of the relative angles shows a fourpeak structure. At $214 \mathrm{~K}$, however, only two peaks appear in the distribution, which indicates a magnetic phase transition to the $1 Q$ phase at higher temperatures.

To identify the different magnetic phases as a function of the temperature, we determined the heat capacity of the $\mathrm{Ru}_{2} \mathrm{MnSi}$ and $\mathrm{Ru}_{2} \mathrm{MnSb}$ Heusler compounds which we present in Fig. 6. In the case of the $\mathrm{Ru}_{2} \mathrm{MnSi}$ system the heat capacity shows two phase transitions [see Fig. 6(a)]. The lowtemperature phase below $200 \mathrm{~K}$ possesses the noncollinear $3 Q$ or $4 Q$ states and the higher-temperature phase corresponds to the $1 Q$ state, with a critical temperature of $T_{\text {crit }}=342 \mathrm{~K}$ above which the system is paramagnetic. Our simulations indicate that the small peak below the transition to the $1 Q$ phase arises due to an instability at which thermal fluctuations are strong enough to induce spontaneous transitions between 
TABLE I. Critical temperatures for $\mathrm{Ru}_{2} \mathrm{MnSb}$ and $\mathrm{Ru}_{2} \mathrm{MnSi}$ alloys in the present work from spin dynamics simulations and in Ref. [15] from Monte Carlo simulations. The experimental transition temperatures are also presents as reported in Ref. [39].

\begin{tabular}{lccc}
\hline \hline & $\begin{array}{c}\text { SD sim. } \\
\text { This work }\end{array}$ & $\begin{array}{c}\text { MC sim. } \\
\text { Ref. [15] }\end{array}$ & $\begin{array}{c}\text { eExp. } \\
\text { Ref. [39] }\end{array}$ \\
\hline $\mathrm{Ru}_{2} \mathrm{MnSb}$ & 256 & 180 & 195 \\
$\mathrm{Ru}_{2} \mathrm{MnSi}$ & 346 & 415 & 313 \\
\hline \hline
\end{tabular}

the $3 Q$ and $4 Q$ configurations. We performed simulations by heating up the system selectively either from the $3 Q$ or from the $4 Q$ state at $T=0 \mathrm{~K}$, and confirmed the existence of the low-temperature phase transition at the same temperature. Remarkably, the peak in the heat capacity at this temperature occurs due to its contribution from the biquadratic couplings in Eq. (1), while the part of the heat capacity related to the Heisenberg couplings behaves smoothly and shows singularity at $T_{\text {crit }}$ only.

For the $\mathrm{Ru}_{2} \mathrm{MnSb}$ system only one phase transition is observed from the heat capacity [see Fig. 6(b)], where the system transforms directly from the noncollinear state into the paramagnetic one. For this alloy the $\mathrm{NN}$ biquadratic coupling is two times larger than in the case of $\mathrm{Ru}_{2} \mathrm{MnSi}$, which explains the absence of the multiple- $Q$ to $1 Q$ phase transition. For both systems the critical temperature differs somewhat from the previously reported values in Ref. [15] due to the different NN isotropic couplings, but the obtained critical temperatures are also in good agreement with the experiment [39], see Table I. The overwhelming similarity of the thermal properties when the $3 Q$ or the $4 Q$ state is selected at low temperature indicates that for both alloys Fig. 6 displays relevant phase transitions even in the case when one of these phases is energetically preferred by higher-order multispin interactions not included in the spin model (1).

\section{CONCLUSION}

In conclusion, we examined the magnetic equilibrium states of the $\mathrm{Ru}_{2} \mathrm{MnZ}(Z=\mathrm{Sn}, \mathrm{Sb}, \mathrm{Ge}, \mathrm{Si}$ ) chemically ordered, full Heusler alloys at zero and finite temperatures within a multiscale simulation approach. We performed first-principles calculations in the paramagnetic state via the scheme of disordered local moments and obtained the isotropic and biquadratic couplings between the $\mathrm{Mn}$ atoms using the spin-cluster expansion technique. We found frustrated bilinear couplings for all considered systems and NN biquadratic coupling that favors noncollinear alignment between the $\mathrm{Mn}$ atoms in the case of $\mathrm{Ru}_{2} \mathrm{MnSi}$ and $\mathrm{Ru}_{2} \mathrm{MnSb}$ compounds, while collinear alignment in case of $\mathrm{Ru}_{2} \mathrm{MnGe}$ and $\mathrm{Ru}_{2} \mathrm{MnSn}$. The frustrated isotropic interactions with the biquadratic coupling lead to a noncollinear antiferromagnetic state in the case of $\mathrm{Ru}_{2} \mathrm{MnSi}$ and $\mathrm{Ru}_{2} \mathrm{MnSb}$ alloys. This noncollinear magnetic ground state comprises energetically equivalent $3 Q$ or $4 Q$ states that occur with equal probability.

We investigated the thermal stability of the noncollinear state and found a transition to the collinear antiferromagnetic order ( $1 Q$ state) around $200 \mathrm{~K}$ for the $\mathrm{Ru}_{2} \mathrm{MnSi}$ alloy. In the case of $\mathrm{Ru}_{2} \mathrm{MnSb}$ the $\mathrm{NN}$ biquadratic coupling was two times larger than in the case of $\mathrm{Ru}_{2} \mathrm{MnSi}$, thus, the noncollinear state was stable against thermal fluctuations and not transformed to the $1 Q$ state below the paramagnetic phase transition.

From powder neutron diffraction experiments a spinreorientation transition from [110] to [111] direction was inferred at about $100 \mathrm{~K}$ [13] for $\mathrm{Ru}_{2} \mathrm{MnSb}$ and a corresponding peak in the magnetization curve of $\mathrm{Ru}_{2} \mathrm{MnSb}$ was also detected in Ref. [39]. In the same work a pronounced peak in the magnetization was found at $25 \mathrm{~K}$ for $\mathrm{Ru}_{2} \mathrm{MnSi}$, but its origin was not clearly understood. Although the existence of the multiple $-Q$ states has not yet been confirmed experimentally in the investigated Heusler alloys, our results indicate the importance of higher-order exchange interactions in the magnetic equilibrium state of the antiferromagnetic materials. We expect that the present work may motivate further experimental investigations of antiferromagnetic Heusler alloys and provide a possible application of the noncollinear antiferromagnetic materials.

\section{ACKNOWLEDGMENTS}

This work was supported by the National Research, Development, and Innovation Office of Hungary under Projects No. PD120917, No. K115575, and No. K131938, as well as by the BME Nanotechnology and Materials Science TKP2020 IE grant of NKFIH Hungary (BME IE-NAT TKP2020). The authors would like to acknowledge NIIF for awarding us access to resources based in Hungary at Debrecen.
[1] T. Jungwirth, J. Sinova, A. Manchon, X. Marti, J. Wunderlich, and C. Felser, Nat. Phys. 14, 200 (2018).

[2] R. Duine, Nat. Mater. 10, 344 (2011).

[3] V. Baltz, A. Manchon, M. Tsoi, T. Moriyama, T. Ono, and Y. Tserkovnyak, Rev. Mod. Phys. 90, 015005 (2018).

[4] H. V. Gomonay and V. M. Loktev, Phys. Rev. B 81, 144427 (2010).

[5] P. Wadley, B. Howells, J. Železný, C. Andrews, V. Hills, R. P. Campion, V. Novák, K. Olejník, F. Maccherozzi, S. S. Dhesi, S. Y. Martin, T. Wagner, J. Wunderlich, F. Freimuth, Y. Mokrousov, J. Kuneš, J. S. Chauhan, M. J. Grzybowski,
A. W. Rushforth, K. W. Edmonds, B. L. Gallagher, and T. Jungwirth, Science 351, 587 (2016).

[6] R. Yanes, E. Simon, S. Keller, B. Nagyfalusi, S. Khmelevsky, L. Szunyogh, and U. Nowak, Phys. Rev. B 96, 064435 (2017).

[7] W. H. Meiklejohn and C. P. Bean, Phys. Rev. 102, 1413 (1956).

[8] J. Nogués and I. K. Schuller, J. Magn. Magn. Mater. 192, 203 (1999).

[9] A. Hirohata, T. Huminiuc, J. Sinclair, H. Wu, M. Samiepour, G. Vallejo-Fernandez, K. O'Grady, J. Balluf, M. Meinert, G. Reiss, E. Simon, S. Khmelevskyi, L. Szunyogh, R. Y. Díaz, U. Nowak, 
T. Tsuchiya, T. Sugiyama, T. Kubota, K. Takanashi, N. Inami, and K. Ono, J. Phys. D 50, 443001 (2017).

[10] M. Acet, E. Duman, E. F. Wassermann, L. Mañosa, and A. Planes, J. Appl. Phys. 92, 3867 (2002).

[11] E. Simon, J. G. Vida, S. Khmelevskyi, and L. Szunyogh, Phys. Rev. B 92, 054438 (2015).

[12] I. Galanakis and E. Şaşığlu, Appl. Phys. Lett. 98, 102514 (2011).

[13] M. Gotoh, M. Ohashi, T. Kanomata, and Y. Yamaguchi, Phys. B 213-214, 306 (1995).

[14] S. Ishida, S. Kashiwagi, S. Fujii, and S. Asano, Phys. B 210, 140 (1995).

[15] S. Khmelevskyi, E. Simon, and L. Szunyogh, Phys. Rev. B 91, 094432 (2015).

[16] H. Kawamura, J. Phys. Condens. Matter 10, 4707 (1998).

[17] C. Lacroix, J. Phys. Soc. Jpn. 79, 011008 (2010).

[18] I. Martin and C. D. Batista, Phys. Rev. Lett. 101, 156402 (2008).

[19] Y. Akagi, M. Udagawa, and Y. Motome, Phys. Rev. Lett. 108, 096401 (2012).

[20] D. Solenov, D. Mozyrsky, and I. Martin, Phys. Rev. Lett. 108, 096403 (2012).

[21] S. Hayami, R. Ozawa, and Y. Motome, Phys. Rev. B 95, 224424 (2017).

[22] P. Kurz, G. Bihlmayer, K. Hirai, and S. Blügel, Phys. Rev. Lett. 86, 1106 (2001).

[23] F. Canepa, M. Napoletano, A. Palenzona, O. Moze, and W. Kockelmann, J. Phys.: Condens. Matter 17, 373 (2005).

[24] R. Takagi, J. S. White, S. Hayami, R. Arita, D. Honecker, H. M. Rønnow, Y. Tokura, and S. Seki, Sci. Adv. 4, eaau3402 (2018).
[25] B. G. Ueland, C. F. Miclea, Y. Kato, O. Ayala-Valenzuela, R. D. McDonald, R. Okazaki, P. H. Tobash, M. A. Torrez, F. Ronning, R. Movshovich, Z. Fisk, E. D. Bauer, I. Martin, and J. D. Thompson, Nat. Commun. 3, 1067 (2012).

[26] S. H. Vosko, L. Wilk, and M. Nusair, Can. J. Phys 58, 1200 (1980).

[27] L. Szunyogh, B. Újfalussy, P. Weinberger, and J. Kollár, Phys. Rev. B 49, 2721 (1994).

[28] R. Zeller, P. H. Dederichs, B. Újfalussy, L. Szunyogh, and P. Weinberger, Phys. Rev. B 52, 8807 (1995).

[29] B. L. Gyorffy, A. J. Pindor, J. Staunton, G. M. Stocks, and H. Winter, J. Phys. F 15, 1337 (1985).

[30] R. Drautz and M. Fähnle, Phys. Rev. B 69, 104404 (2004).

[31] R. Drautz and M. Fähnle, Phys. Rev. B 72, 212405 (2005).

[32] L. Szunyogh, L. Udvardi, J. Jackson, U. Nowak, and R. Chantrell, Phys. Rev. B 83, 024401 (2011).

[33] A. Lyberatos, D. V. Berkov, and R. W. Chantrell, J. Phys.: Condens. Matter 5, 8911 (1993).

[34] U. Nowak, Classical spin models, in Handbook of Magnetism and Advanced Magnetic Materials (John Wiley and Sons, New York, 2007).

[35] J. L. García-Palacios and F. J. Lázaro, Phys. Rev. B 58, 14937 (1998).

[36] J. Moran-Lopez, R. Rodriguez-Alba, and F. Aguilera-Granja, J. Magn. Magn. Mater 131, 417 (1994).

[37] D. Herrmann-Ronzaud, P. Burlet, and J. Rossat-Mignod, J. Phys. C 11, 2123 (1978).

[38] W. L. Roth, Phys. Rev. 110, 1333 (1958).

[39] T. Kanomata, M. Kikuchi, and H. Yamauchi, J. Alloy. Comp. 414, 1 (2006). 